\title{
BMJ Open Feasibility and acceptability study on the use of a smartphone application to facilitate balance training in the ageing population
}

\author{
Enrica Papi (D) , ${ }^{1,2}$ Shin-Yi Chiou, ${ }^{1,3}$ Alison H McGregor ${ }^{1}$
}

To cite: Papi E, Chiou S-Y, McGregor AH. Feasibility and acceptability study on the use of a smartphone application to facilitate balance training in the ageing population. BMJ Open 2020;10:e039054. doi:10.1136/ bmjopen-2020-039054

- Prepublication history and additional material for this paper is available online. To view these files, please visit the journal online (http://dx.doi.org/10. 1136/bmjopen-2020-039054)

Received 02 April 2020 Revised 20 0ctober 2020 Accepted 17 November 2020

D) Check for updates

(C) Author(s) (or their employer(s)) 2020. Re-use permitted under CC BY-NC. No commercial re-use. See rights and permissions. Published by BMJ.

${ }^{1}$ Department of Surgery and Cancer, Faculty of Medicine, Imperial College London,

London, UK

${ }^{2}$ Bioengineering Department, Imperial College London,

London, UK

${ }^{3}$ School of Sport, Exercise and Rehabilitation Sciences, University of Birmingham, Birmingham, UK

Correspondence to

Dr Enrica Papi;

e.papi@imperial.ac.uk

\section{ABSTRACT}

Objectives This study aims to investigate the feasibility and acceptability of using an app-based technology to train balance in the older population.

Design Prospective feasibility study.

Setting The study was conducted in a university setting and participants' homes.

Participants Thirty-five volunteers $\geq 55$ years old were recruited.

Intervention Participants were asked to follow a balance exercise programme 7 days a week for 3 weeks using a phone application. Seventeen participants trained for a further 3 weeks.

Outcome measures Postural sway measures during quiet standing with feet at shoulder width apart and feet together, one leg standing and tandem stance were measured at baseline, and at the end of the 3 and 6 training weeks; the International Physical Activity Questionnaire (IPAQ) assessed participants' physical activity level before training; and app acceptability was recorded using a user experience questionnaire. Results Participants on the 3 and 6-week programme on average completed $20( \pm 5)$ and $38( \pm 11)$ days of training, respectively, and all scored moderate to high on the IPAQ. Between baseline and the 3-week assessments, statistically significant improvements were observed for anteroposterior sway, mediolateral sway, sway area during tandem stance, for anteroposterior sway during one leg standing and for sway area during feet together stance. Improvements were observed at 6 week compared with baseline but those between 3 and 6 weeks were not significant. Based on the questionnaire, participants reported that the app is an appropriate tool for balance training $(77 \%)$, they reported benefits from the training $(50 \%)$ and found it easy to fit it into daily routine $(88 \%)$. Conclusion The high level of adherence and improvements observed in the analysed measures demonstrate the feasibility of using an app to train balance in moderately to highly physically active older participants. This demonstrates that given appropriate tools the older population is positive towards and receptive of digital interventions aimed to improve balance.

\section{INTRODUCTION}

A fall is an event during which a person inadvertently comes to rest on the ground. ${ }^{1}$ Data
Strengths and limitations of this study

- This study introduces the use of a digital app-based technology to train balance in the older population.

- App feasibility was assessed by comparing centre of pressure sway parameters before and after intervention.

- The use of centre of pressure parameters overcomes the subjectivity of clinical balance scales.

- App acceptability was evaluated.

- App effectiveness requires validation against a control group.

- Findings are based on a highly active small sample size $\geq 55$ years old.

from the WHO indicate that $28 \%-35 \%$ of older people ( $\geq 65$ years) fall each year globally, with prevalence increasing with age. Falls can lead to severe consequences such as fractures, spinal cord injury and traumatic brain injury which all have a huge impact on socioeconomic costs; close to $95 \%$ of all hip fractures are caused by falls, $20 \%$ of patients with hip fracture die within a year and $6.9 \%$ within 30 days. $^{2}$ Since population ageing is a global phenomenon, it is important to understand how to prevent and manage falls with a view to maintaining high levels of quality of life in our elderly populations.

The evidence supporting the use of an exercise programme for those at risk of falling is strong ${ }^{3}$ and is endorsed by the WHO. ${ }^{4}$ Moreover, exercising confers mental as well as physical healthcare benefits. ${ }^{5}$ However, compliance with and adherence to exercise programmes in older age groups is problematic jeopardising the related benefits. Gillespie et $a l \mathrm{~s}^{3}$ review suggests that a preventative exercise intervention for older age groups should comprise balance and strength training. Balance deterioration seen with ageing has been associated with risk of falling. ${ }^{67}$ Previous work has shown differences 
in standing balance between fallers and non-fallers: greater postural sway during semitandem stand was observed in fallers compared with non-fallers ${ }^{8}$; time on single leg standing discriminated fallers from non-fallers. ${ }^{9} 10$ Therefore, improving balance through exercise is important; however, how an exercise programme is delivered (selfdirected or supervised), where it is delivered (at home, within a centre) and the exact content are less clear. ${ }^{11} 12$ Consequently, exercise interventions have not been sufficiently implemented into clinical practice or community services. Moreover, Sherrington et $a l^{13}$ have shown that fall prevention was more effective in exercise programmes that challenge balance, have higher exercise dose and do not include a walking programme. This highlights the need for a novel approach to engaging this population in exercises that challenge their balance alongside strength training to prevent falls. Furthermore, there is evidence that mobile apps incorporating behavioural change techniques such as goal setting, behaviour feedback and selfmonitoring can motivate adults to engage with physical activity for a sustained period of time. ${ }^{14-17}$ Therefore, for the training to be effective in the long term we need to develop a simple self-directed assessment of balance to allow for self-monitoring of progression. This will allow older people to monitor their balance and provide a metric to encourage and facilitate long-term engagement with exercise. Without such metrics, the realisation of a fall prevention strategy will be fraught with difficulties.

The emergence of digital technologies and the rise in the use of smartphones in older populations offer new opportunities to engage with this population. ${ }^{18}$ As well as a medium to deliver and support exercise programmes, such technologies can be used to educate patients with a view to changing their beliefs about exercise by providing tools that will grow their confidence and self-efficacy. This can be achieved through the technology feedback by providing goals, markers of improvement and rewards for exercising, ${ }^{19} 20$ while also offering the potential to be used as self-monitoring tools. ${ }^{14}$ Recently, the use of mobile phones and devices to assess balance has been proposed by exploiting the accelerometers and gyroscopes embedded in each device. ${ }^{2122}$ By applying the same concepts, the app used in this study uses the sensors embedded in the device to guide a balance training programme. Therefore, the aim of this study is to explore the feasibility and acceptability of using a digital app-based technology to train balance and strength in the older population. We particularly focused on balance monitoring and we hypothesised that it is feasible to train balance using an app-based technology in older people.

\section{METHODS}

\section{Study population}

Participants were included in the study if they had access to a smartphone or tablet, were $\geq 55$ years old, had no history of falls within a year from study participation and were able to understand and speak English. If participants suffered from irreversible neurological disease or balance disorders (eg, vestibular, visual, somatosensory and proprioceptive conditions), had a current injury to the lower extremity that prevented them from exercising and if their cognitive function prevented them from understanding and undertaking the exercise, they were excluded from the study. Participants were recruited from the local communities via a series of recruitment posters and emails.

\section{Study protocol}

A mobile phone/tablet application (app) (Nymbl, Nymbl Science, Denver, USA) was used to provide participants with a 6 min daily balance exercise training programme. The Nymbl app has been developed using the evidencebased approach as described in the best practice guidelines for the STEADI (Stopping Elderly Accidents, Deaths, and Injuries) protocol, ${ }^{23}$ and this is the first study on its acceptability. Participants downloaded the app during their first study visit and were shown how to use the app by the research team. Moreover, they were required daily access to a mobile phone/tablet to run the training delivered via the app.

The training programme delivered was individualised to each participant's balance ability based on an initial assessment conducted via the app. This initial appbased assessment consisted of a series of balance tests of increasing difficulty of $30 \mathrm{~s}$ each (eg, stand with feet at shoulder width apart, stand with feet together, tandem stand, one-leg stand), the Timed Up and Go (TUG) test and a $30 \mathrm{~s}$ sit-to-stand (STS) task. These were conducted via the app with the participants holding their phone in front of them. The app, using the smartphone's in-built accelerometer, measures the participant's sway during the balance tests stopping the tests if excessive movement occurs. If the participant is able to complete the test, he/ she will be asked by the app to perform the next test of the following level of difficulty. If the participant fails to maintain his/her balance during the $30 \mathrm{~s}$ test, they will move to the TUG test and to the $30 \mathrm{~s}$ STS task. For the TUG test, the app measures the time to complete the task, and for the $30 \mathrm{~s}$ STS task the participant is asked to input the number of STS repetitions performed. Participants' exercise level for the training was determined from this assessment via the app proprietary algorithm. The app offers six levels of exercise intensity (from bronze to gold + ) with the main difference between levels being the duration of each exercise prescribed. The exercises included were a combination of different strengthening exercises (eg, tandem stand, short lunge left/right, stand-ups, wall sit). To enhance the training effect, each exercise had a cognitive component; participants were prompted to answer multiple-choice questions while performing the exercises, thereby creating a dual task challenge for participants which has been associated with greater improvement in balance training. ${ }^{24}$ As participants became proficient at their initial training level, the app would offer them a promotion to the next level of exercise intensity. 
Guidance on how to perform each exercise was delivered by the app, which also provided feedback on exercise execution and tracked their progress. Feedback consisted of a summary on how many exercises were completed successfully and/or the time each exercise was performed if the participant was not able to complete it. Participants' progress and status were saved in an online portal, permitting remote monitoring of the participants thereby allowing us to measure their exercise adherence. The online portal recorded information on the exercises performed each day, the target and actual scores of each exercise and the participant's training level.

All participants followed the balance training programme for either 3 or 6 weeks. Allocation was based on preference and availability at the start of testing. Eighteen participants followed the programme for 3 weeks and 17 participants for 6 weeks.

Moreover, participants attended two (3-week training programme participants) or three (6-week training programme participants) balance laboratory-based assessments: at baseline (Test 1), 3 weeks (Test 2 ) and 6 weeks (Test 3; for 17 participants only). A portable force plate (Kistler Type 9286B, Kistler Instrumente, Winterthur, Switzerland) operating at $1000 \mathrm{~Hz}$ was used to record centre of pressure $(\mathrm{CoP})$ parameters. Participants stood on the force platform with their feet at shoulder width apart, feet together, tandem position and on one leg for $30 \mathrm{~s}$. This trial duration was chosen, since it is consistent with previous studies and would be achievable for most participants during the more difficult balance tasks. ${ }^{2526}$ Each of the above balance tasks was performed once and the same tasks were repeated at each visit. In addition, the International Physical Activity Questionnaire (IPAQ) was recorded to assess participants' physical activity level prior training. ${ }^{27}$ Finally, during their last visit, participants completed a questionnaire (table 1) to provide feedback on their experience of using the app for balance training.

Table 1 Participant's experience questionnaire

Questions Possible answers

1. How important do you believe improving and maintaining your balance to 1 (not at all) to 10 (very much so) be?

2. Do you think the proposed training is a good way of achieving the above? Yes/no/not sure

\section{Overall, how beneficial did you find the training to be?}

4. Have you noticed any other physical benefits or improvements after completing the training?

5. How easy was it to fit the training into your daily routine?

6. Would you continue using the new, improved app when it is available?

7. If you were to keep using the Nymbl app, how regularly do you think you would use it?

8. Which of the following did you enjoy about using the app?

(Tick all that apply.)
1 (not at all) to 10 (very much so)

Yes/no/not sure

If yes, please specify

1 (not at all) to 10 (very much so)

Yes/no/not sure

- Every day

- A couple of times a week

- Just occasionally

- Whenever I felt like my balance needed a boost

- I don't think I would use it again

- Fun to use

- Instructions clear and helpful

- Attractive design

- Functional design

- The 'Man' helped visualise the exercises

- The questions taught me a few things

- Sense of achievement after using it

- Ability to use it anywhere, any time

- Other:....

- Technical difficulties

- Instructions not clear

- Instructions too complicated

- Didn't enjoy the activities

- Lack of variety in questions

- Just forgot

- Didn't feel enough progression through different levels

- Every day was just too often

- Exercises too physically challenging

- Other: 


\section{Data and statistical analysis}

Data analysis was performed with custom code in Matlab (MathWorks, Natick, USA). The last $20 \mathrm{~s}$ of each trial was analysed to remove any transient effects resulting from settling into the required position. ${ }^{25} \mathrm{CoP}$ displacement in the anteroposterior $(\mathrm{A} / \mathrm{P})$ and mediolateral $(\mathrm{M} / \mathrm{L})$ directions was obtained from the force platform and used to calculate the $\mathrm{CoP} \mathrm{A} / \mathrm{P}$ sway, M/L sway, sway velocity and sway area. The $\mathrm{A} / \mathrm{P}$ and $\mathrm{M} / \mathrm{L}$ sways were calculated as the $\mathrm{SD}$ of the $\mathrm{A} / \mathrm{P}$ and $\mathrm{M} / \mathrm{L}$ displacements, respectively, and from the $\mathrm{A} / \mathrm{P}$ and $\mathrm{M} / \mathrm{L}$ displacements the mean sway velocity was computed using a backward difference scheme. The sway area was calculated as the elliptical area that encapsulates $95 \%$ of the sway path derived from the $\mathrm{CoP} \mathrm{A} / \mathrm{P}$ and $\mathrm{M} / \mathrm{L}$ displacements. ${ }^{28}$

Data were tested for normality using the ShapiroWilk test. Depending on data normality, paired t-tests or Wilcoxon signed-rank tests (WSRT) were used to compare force plate measures between the initial and 3-week assessments. Although for the 6-week training group the analysis was exploratory based on a small sample size, comparisons among the three assessments were performed using a repeated measures analysis of variance (ANOVA). If significant differences were observed from the ANOVA analysis post hoc pairwise comparisons with Bonferroni correction were conducted. The significance level was set at 0.05 . Statistical analysis was performed using SPSS (V.24, SPSS).

\section{Patient and public involvement}

Patients were not involved in formulating the study questions or the outcome measures nor were they involved in the design and implementation of the study. Results will be shared with participants in the form of a newsletter.

\section{RESULTS}

Thirty-five participants over the age of 55 took part in the study (mean age: $68 \pm 6.2$ years; height: $168 \pm 9.2 \mathrm{~cm}$; body mass: $67.6 \pm 13$; 10 men, 25 women).

Participants' adherence to the training programme was excellent; those who undertook the training for 3 weeks (21 days) and for 6 weeks (42 days) performed the training for an average of 20 \pm 5 days (range: 13-27 days) and 38 \pm 11 days (range: 11-52 days), respectively. All participants scored moderate (8) to high (26) in the IPAQ, apart from one who scored low. The 3-week cohort and the 6 -week cohort did not differ for age $(p=0.6)$ and they presented with similar balance ability at baseline with the only significant difference found in the CoP velocity during standing with feet together $(\mathrm{p}<0.05)$.

\section{Force platform parameters}

Figure 1A-D shows the bar plots with means and SDs of the four outcomes measured at baseline (Test 1) and 3-week assessment (Test 2) for all tasks performed. Overall, the parameters assessed showed a pattern of improvement whereby A/P sway, M/L sway, sway area and sway velocity changed by $21 \% \pm 15 \%, 8 \% \pm 10 \%, 29 \% \pm 21 \%$ and $8 \% \pm 5 \%$, respectively, between Test 1 and Test 2 across all participants. Statistically significant differences between tests were found for the $\mathrm{A} / \mathrm{P}$ sway during one-leg stand $(-1.5$ $\mathrm{mm} ; \mathrm{p}=0.02$, WSRT $)$ and tandem stand $(-3.2 \mathrm{~mm} ; \mathrm{p}<0.00$, WSRT) (figure 1A). M/L sway significantly differed between tests during feet together $(-0.6 \mathrm{~mm}$; $\mathrm{p}=0.016$, t-test $)$ and tandem stand $(-1.3 \mathrm{~mm} ; \mathrm{p}=0.013$, WSRT) (figure 1B) similar to the sway area (feet together: -128.7 $\mathrm{mm}^{2}, \mathrm{p}=0.013$; tandem stand: $-682.7 \mathrm{~mm}^{2}, \mathrm{p}=0.001$, WSRT; figure 1C). No significant differences were found for sway velocity.

Figure 2A-D shows the four outcomes measured at the baseline (Test 1), 3-week assessment (Test 2) and 6-week assessment (Test 3) for the 17 participants who trained over a 6 -week period. The repeated measures ANOVA showed that the M/L sway differed significantly between time points during the feet together task $(F(2$, $32)=5.030, \mathrm{p}=0.013, \eta_{\mathrm{p}}{ }^{2}=0.2$ ) (figure $2 \mathrm{~B}$ ). Post hoc tests revealed that the $\mathrm{M} / \mathrm{L}$ sway was reduced by an average of $0.91 \mathrm{~mm}$ after 3 weeks $(\mathrm{p}=0.028)$ and by $0.96 \mathrm{~mm}$ after 6 weeks $(\mathrm{p}=0.044)$ compared with baseline. During the feet together task, the sway area differed significantly between time points $\left(F(2,32)=5.417, \mathrm{p}=0.009, \eta_{\mathrm{p}}{ }^{2}=0.2\right)$ as shown by the repeated measures ANOVA (figure 2C). Post hoc tests showed a difference between baseline (Test 1) and 6-week assessment (Test 3) with a reduction of $203.3 \mathrm{~mm}^{2}$ in sway area over 6 weeks $(\mathrm{p}=0.026)$. There was no difference in sway area between baseline and 3-week assessment $(\mathrm{p}>0.05)$.

Repeated measures ANOVA showed significant differences in the $\mathrm{A} / \mathrm{P}$ sway during tandem stand between time points $\left(F(2,32)=7.081, \mathrm{p}=0.009, \eta_{\mathrm{p}}{ }^{2}=0.3\right) \quad($ figure $2 \mathrm{~A})$. Post hoc tests showed differences between Test 1 and Test $2(\mathrm{p}=0.015)$ with a reduction in sway of $3.5 \mathrm{~mm}$ at 3 weeks after training. Between Test 2 and Test 3 there was a significant difference $(p=0.004)$ but with an increase of $2.0 \mathrm{~mm}$ in the A/P sway at Test 3 . There was no difference between Test 1 and Test 3 but the A/P sway at Test 3 was however still smaller by $1.5 \mathrm{~mm}$ when compared with Test 1 .

Overall, a trend of improvement (eg, lower bars) can be observed for the 6-week training group between Test 1 and Test 3 but the improvement is not always evenly achieved between time points, with some tasks/parameters showing better improvement at Test 2 than at Test 3 .

Number values of each bar plot can be found in the online supplemental file 1 .

\section{Participants' experience questionnaires}

Thirty-four participants (97\%) completed the feedback questionnaire. All participants recognised the importance of maintaining a good balance with only one participant scoring 3 out of 10 (1: not at all; 10: very much so); all other participants scored 7 and above. Twenty-four participants $(71 \%)$ indicated that the app was the appropriate way to achieve and maintain good balance with 26 $(77 \%)$ also reporting that they will continue to use the 


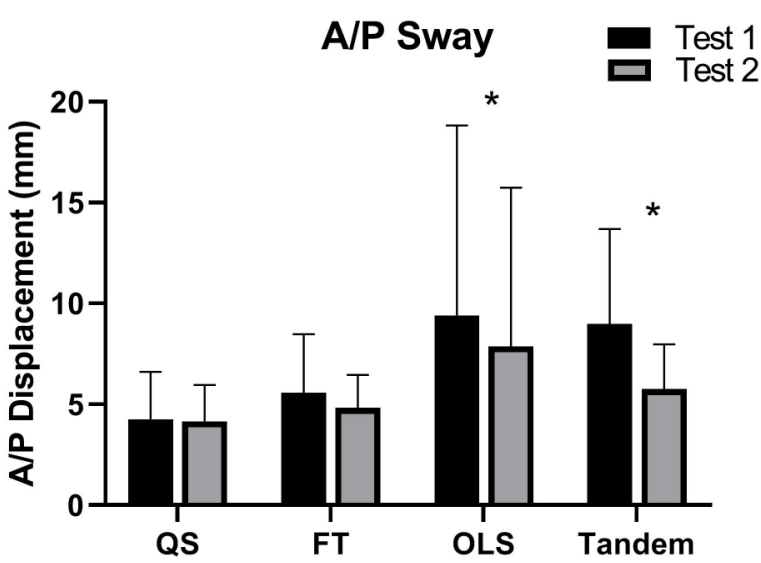

(a)

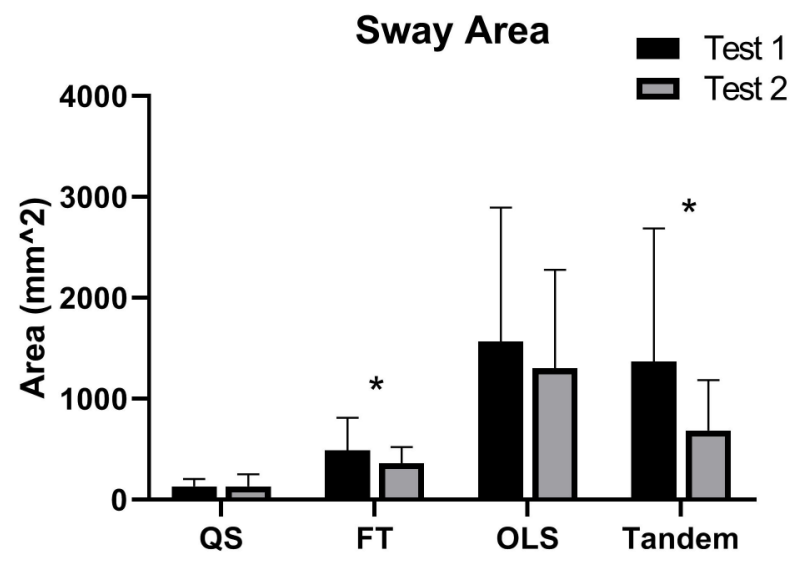

(c)

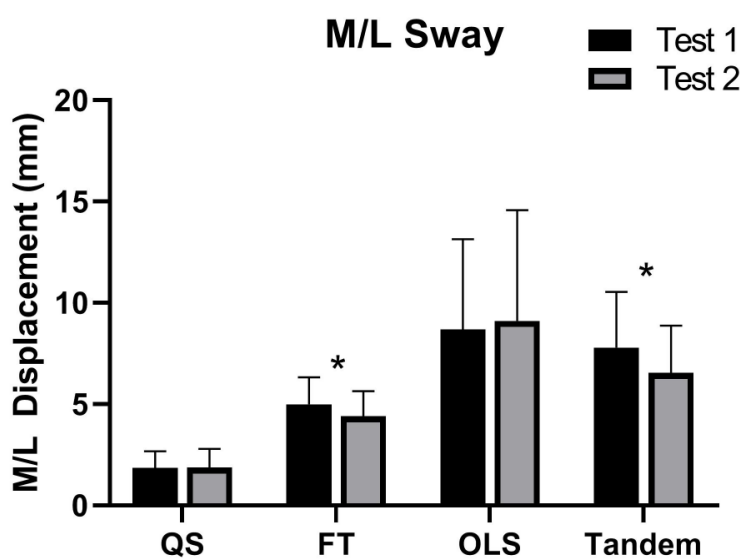

(b)

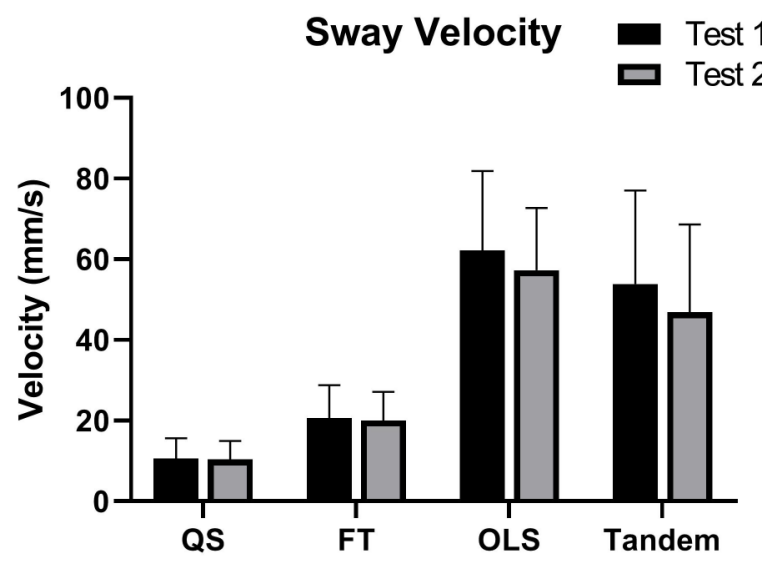

(d)

Figure 1 Bar charts of balance parameters A/P Sway (a), M/L Sway (b), Sway Area (c), Sway Velocity (d), during quiet standing (QS), feet together (FT), one leg standing (OLS) and tandem tasks for baseline (Test 1) and 3-week assessment (Test 2) for all participants $(n=35)$. *Indicates statistically significant difference. A/P, anteroposterior; M/L, mediolateral.

app after the study. Ten participants $(29 \%)$ reported they were uncertain about the use of the app to deliver balance training, and the main reason was that 'the exercises should have been changed'.

The training was extremely beneficial for $50 \%$ of the participants, with 17 scoring 10/10 (figure 3A); only two participants provided a score below 5/10. Sixteen participants $(47 \%)$ reported to have noticed other physical benefits beyond improved balance after training including 'muscle strength' and 'confidence'. However, eight participants (24\%) said no to other perceived benefits and $10(29 \%)$ were not sure.

The majority of participants also found it easy to fit the training in their daily routine, with $88 \%$ of participants scoring it between 6 and 10, and four participants between 5 and 1 (figure 3B). When asked about how regularly they were going to use the app in the future, 20 $(59 \%)$ answered every day, $10(29 \%)$ a couple of times a week, $3(9 \%)$ whenever they felt they needed a balance boost and 1 (3\%) participant said he will not use it.

Figure 4A shows what contributed to app acceptance by participants: key was the sense of achievement (23 participants, 67\%) and guidance provided (23 participants, 67\%: the 'Nymbl Man' helped visualise the exercises; 20 participants, 59\%: instructions clear and helpful). On the other hand, limitations to app use (figure 4B) were the lack of variety within the questions (9 participants, 26\%), forgetfulness (7 participants, 21\%) and poor progression across different exercise levels (4 participants, $12 \%$ ).

\section{DISCUSSION}

The majority of current care for falls focuses on helping people who have already fallen instead of preventing them from falling. The aim of this study was to investigate the feasibility of using a mobile phone/tablet app to train balance among elderly participants and their acceptance, with the view to improving and maintaining good balance to prevent future falls. The findings demonstrate the feasibility of using a phone application to deliver an exercise programme for balance training. This is in line with previous studies reporting feasibility of delivering home-based exercise programme through smartphone 


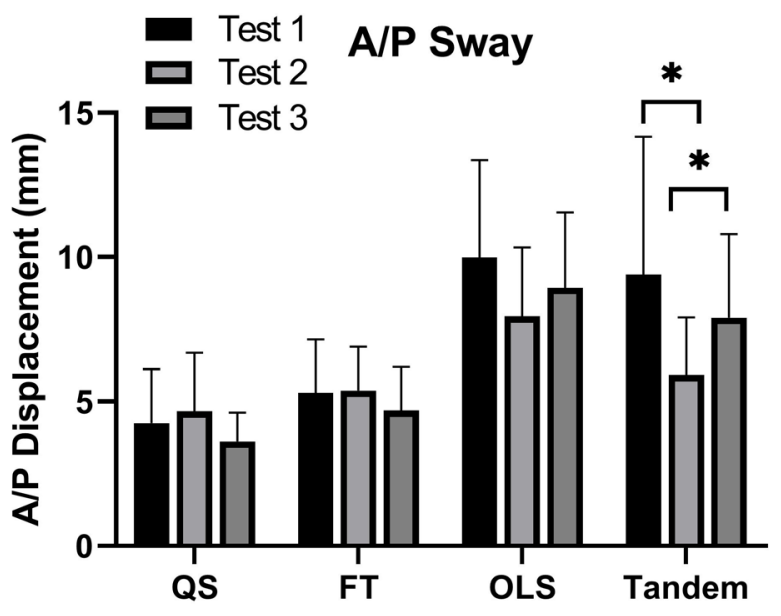

(a)

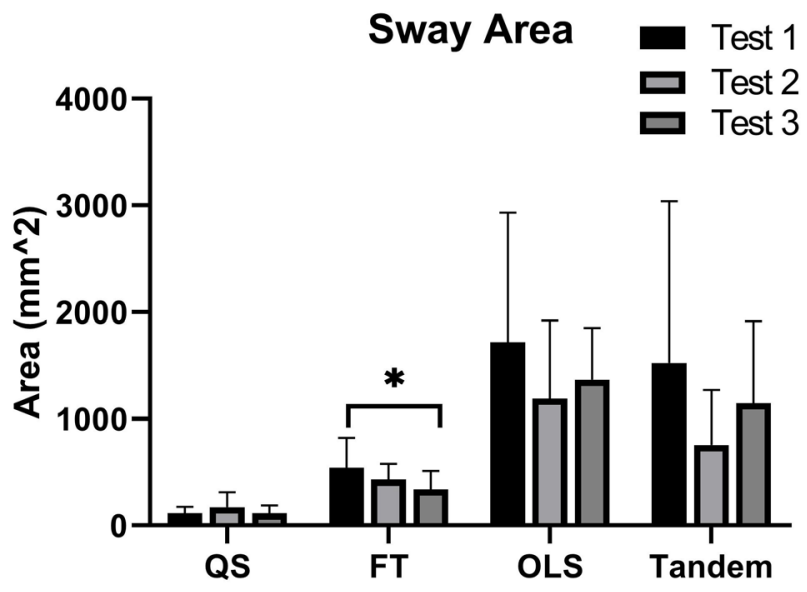

(c)

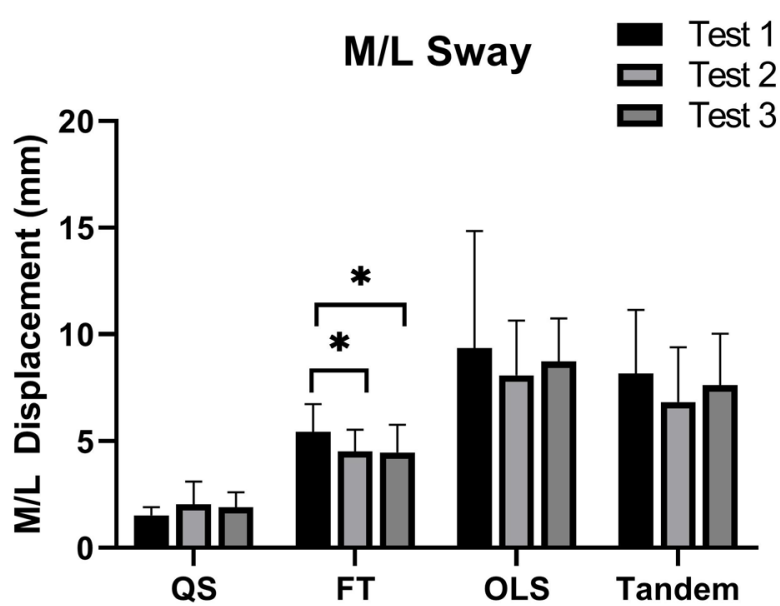

(b)

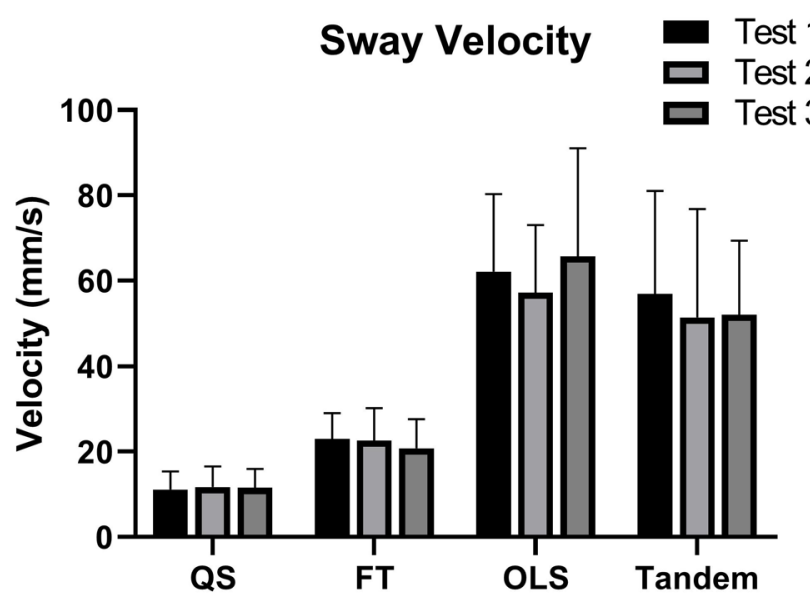

(d)

Figure 2 Bar charts of balance parameters A/P Sway (a), M/L Sway (b), Sway Area (c), Sway Velocity (d), during quiet standing (QS), feet together (FT), one leg standing (OLS) and tandem tasks for baseline (Test 1), 3-week assessment (Test 2) and 6-week assessment for the 6 -week training participants $(n=17)$. *Indicates statistically significant difference. A/P, anteroposterior; M/L, mediolateral.

apps for promoting physical activity in older adults. ${ }^{14} 1520$ Our participants were positive about the app and engaged well with the given exercise programme as shown by the high adherence recorded. Adherence is a major barrier to the effectiveness of prescribed exercise regimes ${ }^{29}$ and the ability to supersede this with the use of an app is powerful. A review indicated that adherence to exercise programmes to prevent falls among older adults is low and further decreasing over time with a reported $44 \%$ adherence at 2 years in a randomised controlled trial. ${ }^{30}{ }^{31}$ Not surprisingly, a Cochrane review into this issue recognised a need for new methods to increase older adults' engagement with exercise. ${ }^{3}$ A previous study reported enhanced adherence in participants who followed an exercise programme delivered by an app when compared with traditional paper handout methods. ${ }^{32}$ This was attributed to the feedback and the guidance provided by the app; also our participants reported an appreciation for the instructions provided and the sense of achievement after use (figure 4A) which may have contributed to the adherence observed. Moreover, in older adults at risk of falling, self-efficacy and lack of confidence in performing exercise and activities of daily living have also been reported to hamper exercise participation and self-management. ${ }^{33-35}$ By providing measures of progress and exercise feedback, the app can promote self-efficacy and confidence similar to social and cognitive-behavioural therapies. This aligns to other studies adopting behaviour change theory in their apps to motivate and empower individuals to adhere to exercise and fall prevention programmes and to sustain exercising over time ${ }^{15-17}$ However, to achieve this, the Nymbl app needs further developments to be able to provide enough variety of exercises to avoid boredom; to be able to tailor exercises to each person's ability; and to be simple and clear (figure 4B).

In addition to participants' positive appreciation and perceived benefits, improvements in postural sway were also observed. Significant differences in sway parameters 


\section{How beneficial did you find the training?}

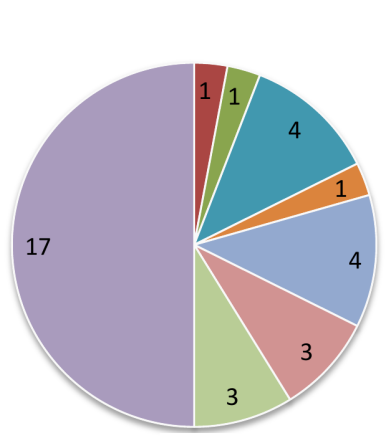

(a)

\section{How easy was to fit the training in daily routine?}

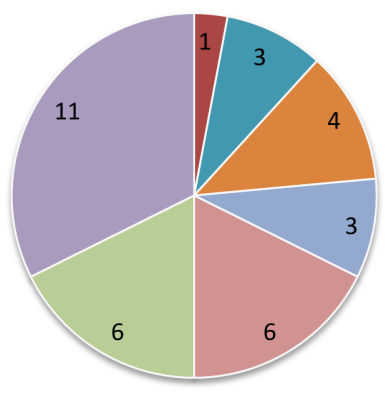

(b)

Figure 3 Pie charts showing participants scoring (1=not at all; $10=$ very much so) to two questionnaire questions: (a) benefit of the training, (b) fit of the training on daily routine.

were observed between baseline and 3-week assessment during the tandem task; however, improvements were also observed in the other tasks (figure 1). The smallest improvements in sway parameters were observed during quiet standing, the simplest of the tasks performed. This is not surprising since the majority of our participants were healthy and physically active. One leg standing showed the biggest variability between participants within each assessment. These findings suggest that to assess and monitor improvement in balance performance the tandem task may be the most informative. A recent review suggested the need of identifying better metrics and tasks to assess balance based on mobile application. ${ }^{22}$ Our findings suggest that the tandem task could be incorporated as an exercise for self-assessment in future app developments. When the intervention was extended to 6 weeks, improvements were still observed from the baseline assessment, some of which were statistically significant (figure 2), but less so when the comparison was made between the 3 and 6 -week assessments. One of the reasons for this could be that the exercises after 3 weeks were not challenging enough given the high level of physicality of our participants. Moreover, 7 out of 17 participants reduced their training days from the first 3 weeks and following 3 weeks by an average of $6( \pm 6)$ days and this could have also affected the results. Nonetheless, a trend of improved sway parameters following the intervention could be appreciated. This aligns with previously conducted studies reporting balance improvements following an exercise intervention. Our results of sway parameters were comparable with that reported in previous studies for similar age groups (ie, $\geq 60$ years old) ${ }^{36}{ }^{37}$ Balance sway as measured by a force platform to quantify balance performance was rarely reported, with studies reporting stance timings or clinical scale scores such as the Tinetti Balance Assessment Score and the Berg Balance Scale. ${ }^{38-40}$ These tools are well suited for clinical practices as they do not require additional instrumentation and can provide quick feedback to the patients but their sensitivity in detecting balance changes may be questionable. ${ }^{41}{ }^{42}$ Therefore, providing a solid measure of postural sway using force platforms or trunk acceleration as commonly conducted in other health contexts will strengthen the evidence of exercise effectiveness in balance performance. ${ }^{43-46}$ The reliability of these measures and their sensitivity to fall risks have been previously demonstrated supporting their use for assessing balance training effectiveness. ${ }^{2646}$

The reason as to why statistical significance was only observed for some parameters could be related to the small sample size, particularly for the 6-week group but may be a result of most participants having high IPAQ scores indicating high functional activity levels and thus leaving less room for improvement and exercises proposed not being challenging enough. The length of the intervention could have also affected the results. Previously published studies on home exercise programmes for fall prevention had interventions of 8 weeks or above, although poor adherence was reported. ${ }^{30}$ Seventy-seven per cent of our participants stated that they would continue to use the app beyond the completion of the study demonstrating that the length of the intervention was not a limiting factor for them and if they were followed up for a longer period of time, further improvements or no deteriorations may have been observed. The training programme proposed via the app expected participants to train every day and included balance-specific exercises. This approach is expected to increase the efficacy of exercise as previously recommended with indication of balance training, balance dose and absence of walking programme as major factors affecting positively exercise outcomes. ${ }^{13}$ To further confirm the effectiveness of the proposed balance programme, an extended intervention period should be considered and a control group receiving exercise with standard handouts should be included in the study. Delbaere $e t a t^{47}$ are currently conducting a longitudinal randomised controlled study into the effectiveness of a tablet-delivered exercise programme to prevent falls in people over 70 years old. The programme, however, in contrast to the Nymbl app requires a longer training session and the use of specific equipment, in addition to a tablet, which reduces its portability and the ease to fit to daily routine wherever the user is potentially compromising adherence and hence effectiveness. A recent systematic review attempted to define balance exercises and their optimal prescription with respect to frequency, 


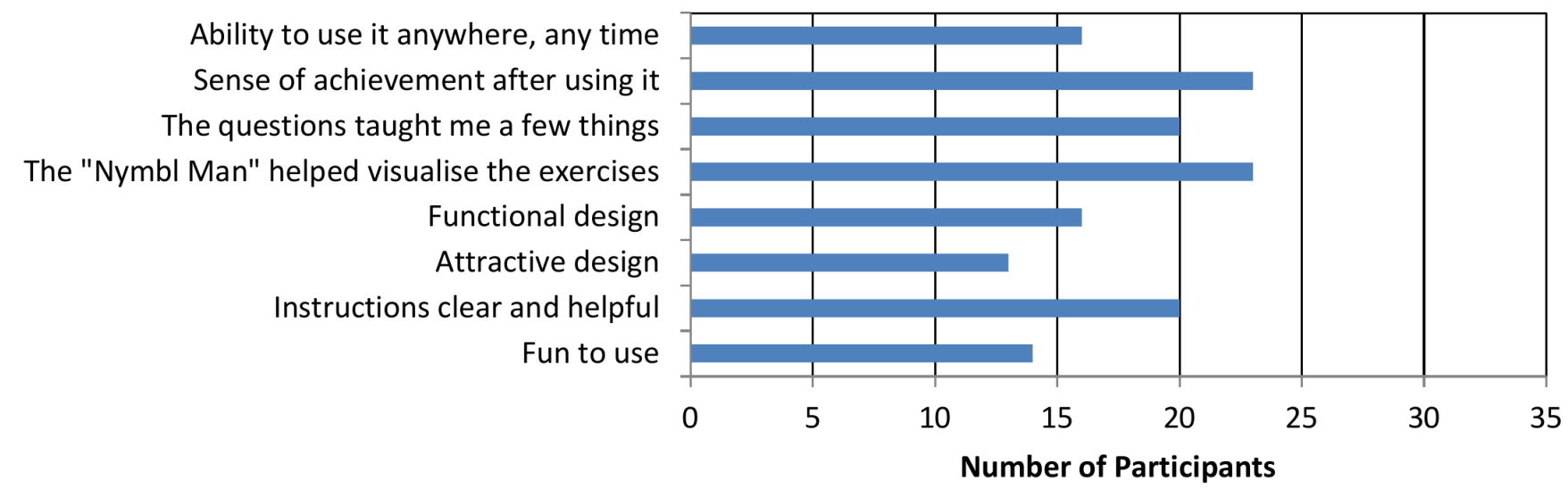

(a)

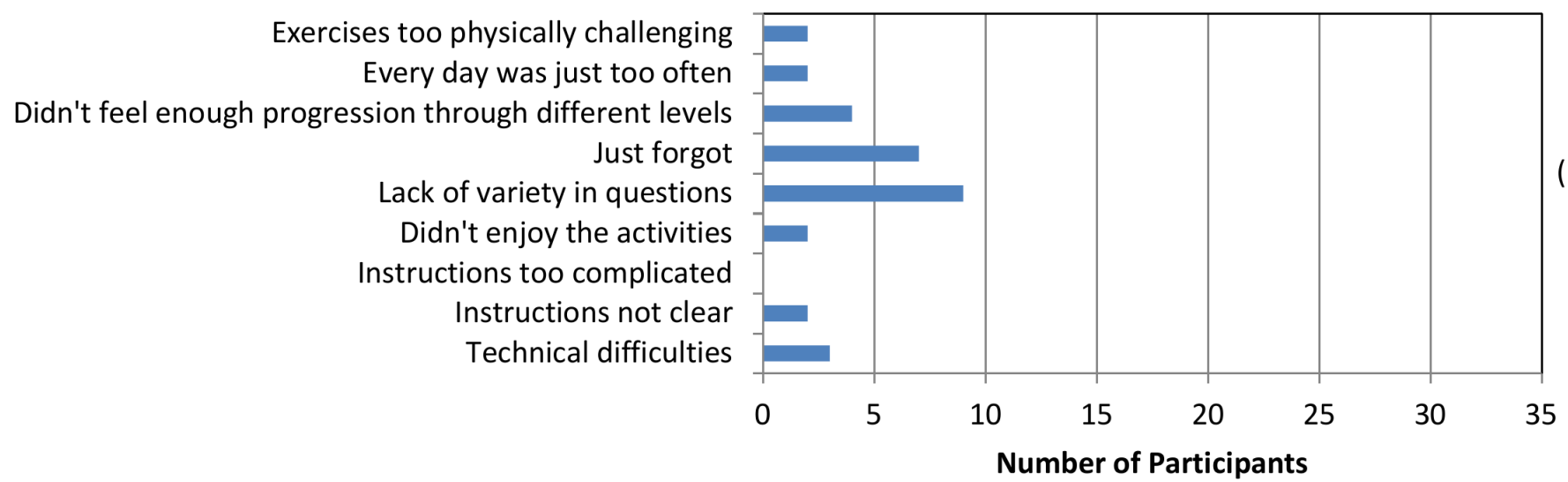

Figure 4 (a) Positive and (b) negative feedbacks provided by participants on the use of the app.

intensity, type, duration and their effect on balance metrics. ${ }^{48}$ Their intervention sessions were of longer duration than the current study but their meta-analyses suggested that exercise intensity was important. The impact of compliance or adherence to exercise was not addressed. Clearly, we need to understand the impact, duration and intensity of the exercise further, as well as consider the ability of the intervention to grow the participants' confidence, an area that to date has received less attention.

One of the limitations of this study is the small sample size particularly for the 6-week training group. As this is a feasibility study a formal sample size calculation has not been performed; however, the sample size was guided by published recommendations, ${ }^{49}{ }^{50}$ and was similar to that used in previous studies.9 ${ }^{9} 112430363945$ Moreover, with regard to participants' experience and views, results are based on a questionnaire with multiple-choice answers which was not underpinned by any particular approach or framework (eg, technology acceptance model). Hence, having further qualitative data may have provided more extensive information. We have also conducted focus groups with current study participants and results will be published as a separate paper. Our adherence results are based on a 6-week maximum training period and longer training should be considered in the future. Finally, participants may have felt an app use bias by participating in the study to not disappoint the research team that could have affected our adherence results.

In conclusion, this study demonstrates the feasibility and acceptability of using a mobile application to train balance among elderly participants. A high level of adherence was recorded, and participants were positive about the use of a phone app to guide their balance training. Improvements were observed in sway parameters although modest; this could be explained by the high level of physicality of our cohort and by the short duration of the intervention. Further studies are necessary to demonstrate the clinical effectiveness of the app, and improvements of the app are necessary to match participants' preferences to maintain adherence. Nonetheless, this approach has the potential to have an effect on practices and policies of healthcare delivery for older adults in a foreseeable future.

Twitter Enrica Papi @enrica_papi

Acknowledgements We would like to thank MSc student Gregoire Aldebert for his contribution with data collection and Denise Lin for her contribution to data processing.

Contributors EP, SYC and AHM conceived and designed the study. EP and SYC carried out the assessments. EP analysed the data and drafted the manuscript. All authors read, edited and approved the final version of the manuscript. All authors had full access to all of the data in the study and can take responsibility for the integrity of the data and the accuracy of the data analysis.

Funding This research received funding from Nymbl. 
Competing interests The authors report grants from Nymbl (Nymbl Science, Denver, USA) during the conduct of the study.

Patient consent for publication Not required.

Ethics approval The study was reviewed and approved by the East MidlandsNottingham 2 Research Ethics Committee (reference number: 17/EM/0042). All participants gave written informed consent.

Provenance and peer review Not commissioned; externally peer reviewed.

Data availability statement Data are available upon reasonable request. Data will be available upon request.

Supplemental material This content has been supplied by the author(s). It has not been vetted by BMJ Publishing Group Limited (BMJ) and may not have been peer-reviewed. Any opinions or recommendations discussed are solely those of the author(s) and are not endorsed by BMJ. BMJ disclaims all liability and responsibility arising from any reliance placed on the content. Where the content includes any translated material, BMJ does not warrant the accuracy and reliability of the translations (including but not limited to local regulations, clinical guidelines, terminology, drug names and drug dosages), and is not responsible for any error and/or omissions arising from translation and adaptation or otherwise.

Open access This is an open access article distributed in accordance with the Creative Commons Attribution Non Commercial (CC BY-NC 4.0) license, which permits others to distribute, remix, adapt, build upon this work non-commercially, and license their derivative works on different terms, provided the original work is properly cited, appropriate credit is given, any changes made indicated, and the use is non-commercial. See: http://creativecommons.org/licenses/by-nc/4.0/.

ORCID iD

Enrica Papi http://orcid.org/0000-0003-0282-7223

\section{REFERENCES}

1 World Health Organization,. Report on falls prevention in older age, 2019. Available: https://www.who.int/violence_injury_prevention/ other_injury/falls/en/

2 Royal College of Physicians. National hip fracture database annual report 2018, 2019. Available: https://www.nhfd.co.uk/files/ 2018ReportFiles/NHFD-2018-Annual-Report-v101.pdf

3 Gillespie LD, Robertson MC, Gillespie WJ, et al. Interventions for preventing falls in older people living in the community. Cochrane Database Syst Rev 2012;9:CD007146.

4 World Health Organization. Falls fact sheets, 2019. Available: https:// www.who.int/news-room/fact-sheets/detail/falls

5 Abdin S, Welch RK, Byron-Daniel J, et al. The effectiveness of physical activity interventions in improving well-being across office-based workplace settings: a systematic review. Public Health 2018;160:70-6.

6 Vieira ER, Palmer RC, Chaves PHM. Prevention of falls in older people living in the community. BMJ 2016;353:i1419.

7 Ganz DA, Bao Y, Shekelle PG, et al. Will my patient fall? JAMA 2007;297:77-86.

8 Doheny EP, McGrath D, Greene BR, et al. Displacement of centre of mass during quiet standing assessed using accelerometry in older fallers and non-fallers. Annu Int Conf IEEE Eng Med Biol Soc 2012;2012:3300-3.

9 Chang C-J, Chang Y-S, Yang S-W. Using single leg standing time to predict the fall risk in elderly. Annu Int Conf IEEE Eng Med Biol Soc 2013;2013:7456-8

10 Macrae PG, Lacourse M, Moldavon R. Physical performance measures that predict faller status in community-dwelling older adults. J Orthop Sports Phys Ther 1992;16:123-8.

11 Otero M, Esain I, González-Suarez Ángel M, et al. The effectiveness of a basic exercise intervention to improve strength and balance in women with osteoporosis. Clin Interv Aging 2017;12:505-13.

12 Lacroix A, Hortobágyi T, Beurskens R, et al. Effects of supervised vs. unsupervised training programs on balance and muscle strength in older adults: a systematic review and meta-analysis. Sports Med 2017;47:2341-61.

13 Sherrington C, Whitney JC, Lord SR, et al. Effective exercise for the prevention of falls: a systematic review and meta-analysis. $J \mathrm{Am}$ Geriatr Soc 2008;56:2234-43.

14 Fukuoka Y, Kamitani E, Bonnet K, Lindgren T, et al. Real-Time social support through a mobile virtual community to improve healthy behavior in overweight and sedentary adults: a focus group analysis. J Med Internet Res 2011;13:e49.
15 King AC, Hekler EB, Grieco LA, et al. Harnessing different motivational frames via mobile phones to promote daily physical activity and reduce sedentary behavior in aging adults. PLOS One 2013;8:e62613.

16 Hawley-Hague $\mathrm{H}$, Tacconi C, Mellone S, et al. Can smartphone technology be used to support an effective home exercise intervention to prevent falls amongst community dwelling older adults?: the together feasibility RCT study protocol. BMJ Open 2019;9:e028100.

17 Taraldsen K, Mikolaizak AS, Maier AB, et al. Protocol for the PreventIT feasibility randomised controlled trial of a lifestyleintegrated exercise intervention in young older adults. BMJ Open 2019;9:e023526.

$18 \mathrm{Kim} \mathrm{H}-\mathrm{S}$, Lee K-H, Kim H, et al. Using mobile phones in healthcare management for the elderly. Maturitas 2014;79:381-8.

19 Rabin C, Bock B. Desired features of smartphone applications promoting physical activity. Telemed J E Health 2011;17:801-3.

20 Vankipuram M, McMahon S, Fleury J. ReadySteady: APP for accelerometer-based activity monitoring and wellness-motivation feedback system for older adults. AMIA Annu Symp Proc 2012;2012:931-9.

21 Pinho AS, Salazar AP, Hennig EM, et al. Can we rely on mobile devices and other gadgets to assess the postural balance of healthy individuals? A systematic review. Sensors 2019;19. doi:10.3390/ s19132972. [Epub ahead of print: 05 Jul 2019].

22 Roeing KL, Hsieh KL, Sosnoff JJ. A systematic review of balance and fall risk assessments with mobile phone technology. Arch Gerontol Geriatr 2017;73:222-6.

23 Stevens JA, Phelan EA. Development of STEADI: a fall prevention resource for health care providers. Health Promot Pract 2013;14:706-14.

24 Ghai S, Ghai I, Effenberg AO. Effects of dual tasks and Dual-task training on postural stability: a systematic review and meta-analysis. Clin Interv Aging 2017;12:557-77.

25 Prieto TE, Myklebust JB, Hoffmann RG, et al. Measures of postural steadiness: differences between healthy young and elderly adults. IEEE Trans Biomed Eng 1996;43:956-66.

26 Swanenburg J, de Bruin ED, Uebelhart D, et al. Falls prediction in elderly people: a 1-year prospective study. Gait Posture 2010;31:317-21.

27 IPAQ. International physical activity questionnaire, 2019. Available: www.ipaq.ki.se

28 Schubert P, Kirchner M. Ellipse area calculations and their applicability in posturography. Gait Posture 2014;39:518-22.

29 Jack K, McLean SM, Moffett JK, et al. Barriers to treatment adherence in physiotherapy outpatient clinics: a systematic review. Man Ther 2010;15:220-8.

30 Simek EM, McPhate L, Haines TP. Adherence to and efficacy of home exercise programs to prevent falls: a systematic review and meta-analysis of the impact of exercise program characteristics. Prev Med 2012;55:262-75.

31 Campbell AJ, Robertson MC, Gardner MM, et al. Falls prevention over 2 years: a randomized controlled trial in women 80 years and older. Age Ageing 1999;28:513-8.

32 Lambert TE, Harvey LA, Avdalis C, et al. An APP with remote support achieves better adherence to home exercise programs than paper handouts in people with musculoskeletal conditions: a randomised trial. J Physiother 2017;63:161-7.

33 Horne M, Skelton DA, Speed S, et al. Falls prevention and the value of exercise: salient beliefs among South Asian and white British older adults. Clin Nurs Res 2014;23:94-110.

34 Fletcher PC, Hirdes JP. Restriction in activity associated with fear of falling among community-based seniors using home care services. Age Ageing 2004;33:273-9.

35 Li F, Fisher KJ, Harmer P, et al. Falls self-efficacy as a mediator of fear of falling in an exercise intervention for older adults. $J$ Gerontol $B$ Psychol Sci Soc Sci 2005;60:P34-40.

36 Ahmad I, Noohu MM, Verma S, et al. Effect of sensorimotor training on balance measures and proprioception among middle and older age adults with diabetic peripheral neuropathy. Gait Posture 2019;74:114-20.

37 Pirouzi S, Motealleh AR, Fallahzadeh F, et al. Effectiveness of treadmill training on balance control in elderly people: a randomized controlled clinical trial. Iran J Med Sci 2014;39:565-70.

38 Haines TP, Russell T, Brauer SG, et al. Effectiveness of a video-based exercise programme to reduce falls and improve health-related quality of life among older adults discharged from Hospital: a pilot randomized controlled trial. Clin Rehabil 2009;23:973-85.

39 Yates SM, Dunnagan TA. Evaluating the effectiveness of a homebased fall risk reduction program for rural community-dwelling older adults. J Gerontol A Biol Sci Med Sci 2001;56:M226-30. 
40 Vogler CM, Sherrington C, Ogle SJ, S.J. Ogle, S.R, et al. Reducing risk of falling in older people discharged from Hospital: a randomized controlled trial comparing seated exercises, weightbearing exercises, and social visits. Arch Phys Med Rehabil 2009;90:1317-24.

41 Allen NE, Canning CG, Sherrington C, et al. The effects of an exercise program on fall risk factors in people with Parkinson's disease: a randomized controlled trial. Mov Disord 2010;25:1217-25.

42 Lin M-R, Wolf SL, Hwang H-F, et al. A randomized, controlled trial of fall prevention programs and quality of life in older fallers. $J \mathrm{Am}$ Geriatr Soc 2007;55:499-506.

43 Mancini M, Horak FB, Zampieri C, et al. Trunk accelerometry reveals postural instability in untreated Parkinson's disease. Parkinsonism Relat Disord 2011;17:557-62.

44 Duffell LD, Southgate DFL, Gulati V, et al. Balance and gait adaptations in patients with early knee osteoarthritis. Gait Posture 2014;39:1057-61.

45 Helbostad JL, Sletvold O, Moe-Nilssen R. Effects of home exercises and group training on functional abilities in home-dwelling older persons with mobility and balance problems. A randomized study. Aging Clin Exp Res 2004;16:113-21.
46 Ruhe A, Fejer R, Walker B. The test-retest reliability of centre of pressure measures in bipedal static task conditions - A systematic review of the literature. Gait Posture 2010;32:436-45.

47 Delbaere K, Valenzuela T, Woodbury A, et al. Evaluating the effectiveness of a home-based exercise programme delivered through a tablet computer for preventing falls in older communitydwelling people over 2 years: study protocol for the Standing Tall randomised controlled trial. BMJ Open 2015;5:e009173.

48 Farlie MK, Robins L, Haas R, et al. Programme frequency, type, time and duration do not explain the effects of balance exercise in older adults: a systematic review with a meta-regression analysis. $\mathrm{Br} \mathrm{J}$ Sports Med 2019;53:996-1002.

49 Billingham SAM, Whitehead AL, Julious SA. An audit of sample sizes for pilot and feasibility trials being undertaken in the United Kingdom registered in the United Kingdom clinical research network database. BMC Med Res Methodol 2013;13:104.

50 Lancaster GA, Dodd S, Williamson PR. Design and analysis of pilot studies: recommendations for good practice. J Eval Clin Pract 2004;10:307-12. 